\title{
The effect of gap depth on the perception of whether a gap is crossable
}

\author{
YANG JIANG and LEONARD S. MARK \\ Miami University, Oxford, Ohio
}

\begin{abstract}
Four experiments were performed in order to examine the effect of gap depth on human observers' perception of whether or not a gap is crossable. Experiments 1 and 2 showed that as the gap's depth increased, observers tended to increasingly underestimate the maximum width of a gap they could step across. Experiments 3 and 4 clarified this finding: The observed covariation of perceived gap crossability and gap depth depended on the observer's direction of gaze, rather than on the physical depth of the gap. The optical relations to which observers might be attending are discussed, as well as the possibility that cognitive-affective processes might have contributed to observers' underestimation of their actual capabilities.
\end{abstract}

When standing at the edge of a gap, how does a person decide whether the gap can be stepped over? The basic environmental constraint on whether a gap is crossable is its width-the distance between the edges of two surfaces of support. The gap's depth does not affect a person's actual stepping capability. However, there are reasons to believe that gap depth may be a factor in an observer's judgment of whether a gap affords stepping across.

The consequences to the actor of (accidentally) falling into a gap, while attempting to step across, change as the gap's depth increases. For the actor who falls into a shallow gap, there will probably be little adverse effect-at worst, wounded pride or a sprained ankle. As depth increases, the consequences become increasingly more dire, with the possibility of broken bones, serious injury, even death. Actors may want to allow a greater margin of safety, thus factoring gap depth into their assessment of gap crossability.

Gap depth may also enter into the perception of a gap's width. A gap is a break in the ground plane with important consequences for both locomotion and perception. In order to determine whether the gap affords stepping over, ${ }^{1}$ observers have to judge the distance from the edge of the surface of support on which they are standing to the edge of the opposing cliff. The essential environmental constraint that has to be perceived, gap width,

This research was supported by a grant from the Committee on Faculty Research at Miami University to L.S.M. Part of this investigation was completed by the first author in partial fulfillment of the requirements for the master's degree at Miami University. The authors acknowledge the assistance of Dawn Anderson, Aaron Domm, Cheryl Baldwin Stewart, Amy Croucher, Natalia Pane, and Mary Beth Scott in the data collection and of Greg Burton, Marvin Dainoff, Michael Turvey, and Rik Warren for their critiques throughout the course of this study, as well as the comments of Myron Braunstein and two anonymous reviewers. Correspondence concerning this article should be addressed to L. S. Mark, Department of Psychology, Miami University, Oxford, OH 45056 (e-mail: lm24psyf@miamiu.acs.muohio.edu). refers to this distance over physically empty space. The edge of the far cliff is defined by the intersection of the ground plane and the vertical wall of the cliff. Although empty space separates the two surfaces of support, the observer's visual field is continuously filled by, from top to bottom, the sky, the ground plane of the opposing cliff, the vertical wall of the opposing cliff, the bottom of the gap, and even the surface on which the observer is standing. Moreover, the edge of the opposing cliff is not simply specified, as, say, a black line on a white background. Its specification is a complex matter, depending on lighting conditions, the textural composition of the ground plane and vertical wall, and the angle at which the two surfaces meet. Since the very existence of the edge of the opposing cliff, to which distance is being judged, depends on both the ground plane and the vertical wall, the optical relationships used to judge gap crossability might reasonably be expected to include the gap's depth.

Finally, there is empirical evidence to support the involvement of gap depth when one is assessing the possibilities for action in crossing a gap. Lock and Collett $(1979,1980)$ examined the actions of toads, Bufo bufo, in negotiating chasms of different depths and widths to obtain food. As expected, for each gap depth the percentage of trials on which the toad leaped across the gap decreased as gap width increased. For a narrow $(7.5-\mathrm{cm})$ gap, the likelihood that the toad would jump across the gap, as opposed to jump into the gap, increased as gap depth increased. Apparently, gaps deeper than $10 \mathrm{~cm}$ need to be jumped across rather than jumped into (and out of ). Most importantly, for a wide gap $(25 \mathrm{~cm})$, which the toads apparently had the ability to leap over, the likelihood that toads would turn away from the gap increased as gap depth increased. Although Lock and Collett's data do not point toward a specific optical relationship between gap depth and width to which the toads attend, their findings showed that the toad's choice of action in "crossing the gap to eat a fly" depended on 
an environmental property, gap depth, that was not germane to the (physical) act of "leaping across the gap." Thus, both a gap's width and its depth may be determinants of whether the gap is crossable.

The current investigation of how observers determine whether a gap can be stepped across began by examining whether judgments of gap crossability by human observers also covary with gap depth. The outcome of the experiments reported here will constrain later attempts to identify the relevant optical relationships that observers use to determine gap crossability. In the first experiment, observers stood at the edge of a gap and estimated the maximum gap width that they could step across for gaps of four different depths.

\section{EXPERIMENT 1}

\section{Method}

\section{Subjects}

Sixteen female undergraduates at Miami University participated in the experiment to satisfy a course requirement.

\begin{abstract}
Apparatus
The gap was constructed using a 152-cm-high, nonmovable platform on which subjects stood, and a movable platform on wheels (Figure 1).

Observers stood on the platform, which had a $122 \times 61 \mathrm{~cm}$ surface. The movable part of the gap had a $115 \times 76 \mathrm{~cm}$ surface. The bottom of the gap was simulated by suspending a large sheet of Styrofoam under the two platforms (Figure 1). The Styrofoam could be set at four different heights, which made four optical depths for the gap: $14,46,92$, and $146 \mathrm{~cm}$. Since the movable platform had an open back, the Styrofoam could pass through it as the movable platform approached the observer. For each gap depth, the distance between the gap edge and the Styrofoam as well as the top of the cliff (horizontal surface) was covered by a piece of burlap. The $14-\mathrm{cm}$ and $46-\mathrm{cm}$ gaps were lower than the $50 \mathrm{th}$ percentile knee height $(49.8 \mathrm{~cm})$ for the American female; the $92-\mathrm{cm}$ was slightly higher than the 50 th percentile leg length $(83.5 \mathrm{~cm})$ for the American female; $146 \mathrm{~cm}$ was slightly lower than the $50 \mathrm{th}$ percentile eye height $(148.9 \mathrm{~cm})$ for the American female (Kroemer \& Price, 1982). The mean eye height of the 16 participants was $155.8 \mathrm{~cm}$.

A $183-\mathrm{cm}$-high screen was placed at the back of the movable platform to hide the experimenter, who stood behind the apparatus. The movable platform could be moved smoothly by the experimenters in the range of $0-200 \mathrm{~cm}$ from the stationary platform. Because of the total height of an observer standing on the platform, the apparatus had to be located in a stairwell with a high ceiling. A curtain prevented the observer from seeing the stairs.
\end{abstract}

\section{Procedure and Experimental Design}

A one-factor (depth), within-subjects design was employed. The subjects were tested individually. All subjects made judgments of maximum crossable gap for each of the four depths. The order of presentation for the four depths was counterbalanced among subjects.

Instructions. The subject observers were carefully instructed regarding both the type of action and the criteria on which we wanted them to base their judgments of gap crossability. The specific style of action on which we wanted observers to base their judgments was defined as follows: (1) The actor must transfer herself completely from one side of the gap to the other side by moving one leg followed by the other leg. (2) The heel of the lead foot should strike the ground first. This means that the entire lead foot

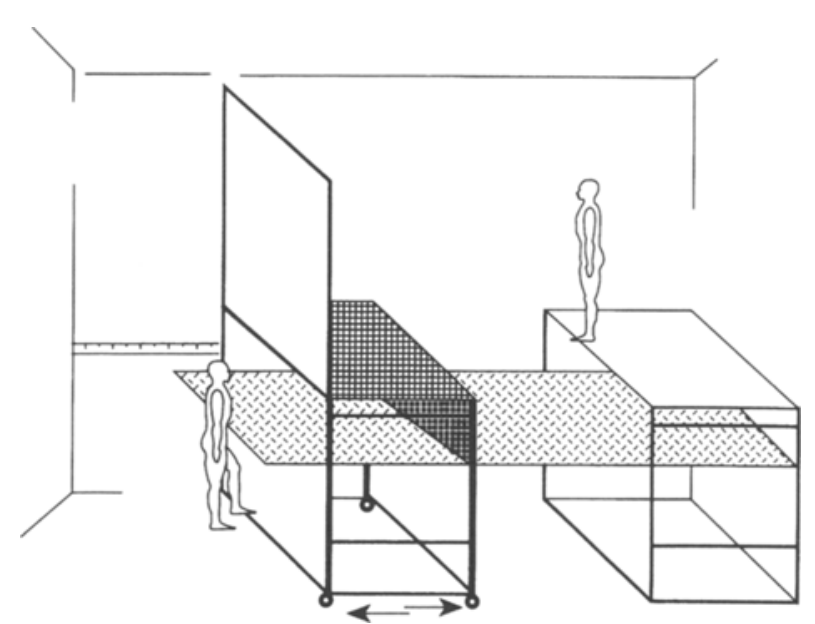

Figure 1. The apparatus used in Experiment 1. The experimenter was hidden from view of the observer.

should be in contact with the ground. (3) One foot should be in contact with the ground at all times. (4) The observers were told that we did not mean crossing the gap either by leaping or jumping. (5) Finally, the observers were told to think about the action as if they were always leading with the same leg.

The observers were instructed to judge the maximum gap width that they felt "confident" they could "just step over safely" by using the prescribed style of action. The instructions emphasized that while we were interested in the widest gap width that they could step across in the specified manner, we also wanted them to be confident that they could complete the action successfully and safely-that is, without falling forward upon landing or slipping backward into the gap. This latter point was reiterated if an observer had any question about our criteria for judging whether a gap was crossable.

Measures of perceived crossable gap size. The subjects stood upright, keeping their toes pointed toward the edge of the platform. Both feet were near the edge of the platform, resting completely on the surface. At no time during the course of the experiment did the observers actually step across the gap. They made all their judgments while they were standing on the $152-\mathrm{cm}$-high platform. The other side of the gap was moved by the experimenters.

Perceived crossable gap size was determined by using a method of limits with four descending trials (i.e., the experimenter pushed the movable side of the gap toward the subject) and four ascending trials (the platform was moved away from the subject) alternately. The subjects were instructed to say "stop" when they felt that the gap width was the maximum distance they could step across safely. They were then given a chance to have the apparatus moved either closer or farther until they were completely satisfied. After making eight judgments at a given depth, the observers were asked to estimate the depth of the gap in familiar units, inches. They were then asked to turn around and face the wall behind them while the experimenters adjusted the depth of the gap.

After making crossability judgments for the four gap depths, the observers stepped down from the platform, walked to a nearby room, and made judgments of how far they could step along the ground. They stood at a line, while one of the experimenters moved a flat, plywood board back and forth in a manner similar to the movement of the platform. The task and procedures used to obtain the observers' judgments of how far they could step on the ground were otherwise identical to those employed earlier. Perceptual judgments on the ground were not counterbalanced with the judgments on the apparatus; we were concerned that having 
some observers make judgments on the ground prior to stepping on the gap apparatus might encourage them to pay more attention to their step length as they were walking down the hall to the apparatus.

Measures of actual stepping capability. After making perceptual judgments of their step length on the ground, the observers were asked to step as far forward as they could on the floor by using the act described by our definition of stepping. They were given five attempts, and they were told to use the same leading leg on each attempt. Step length was measured from the most forward portion of the trailing foot to the heel of the leading foot. Our measure of each person's actual stepping capability was determined by averaging five measurements of her maximum step on the ground.

Anthropometric measures. The experimenters measured each subject's total standing height, eye height, and sitting height. Total leg length was calculated by taking the difference between standing height and sitting height.

\section{Results and Discussion}

The mean actual stepping distance was $101 \mathrm{~cm}(0.65$ of the participants' mean eye height ${ }^{2} ; S D=14.6 \mathrm{~cm}$ ). On the average, the observers underestimated their actual stepping capabilities when making judgments while standing on the ground $(M=93.1 \mathrm{~cm}, 0.60$ of mean eye height; $S D=16.2 \mathrm{~cm}$ ). That the observers underestimated their actual capabilities for all depths, even while standing on the ground, may reflect a general tendency for them to be conservative in estimating their actual capabilities. This tendency could be useful by preventing observers from undertaking an action that they could not successfully complete and that could thus result in injury (Mark, 1987). In view of the fact that fewer than 3\% of all judgments exceeded an individual's actual capability for stepping across the gap, the observers evidently followed our instructions to set the gap width to a distance that they were confident they could step over safely.

Figure 2 shows the mean perceived maximum crossable gap width for each gap depth. Judgments for 13 of the 16 observers showed this tendency to increasingly underestimate their capabilities as gap depth increased. Only 1 observer perceived her capability to increase from $14-$ to $146-\mathrm{cm}$ depths. A one-factor, within-subjects analysis of variance (ANOVA) revealed a significant effect of depth $[F(3,45)=23.36, p<.01]$. A Tukey HSD test $(\alpha=.05)$ indicated that for the 14-cm gap, the mean maximum crossable gap distance, $90.6 \mathrm{~cm}$, was significantly greater than that for the $46-\mathrm{cm}$ gap, $85.5 \mathrm{~cm}$. The mean for the $92-\mathrm{cm}$ gap, $82.5 \mathrm{~cm}$, was significantly greater than that for the $146-\mathrm{cm}$ gap, $77.8 \mathrm{~cm}$. Differences in judgments of maximum crossable gap width between gaps of 46- and 92-cm depths did not reach statistical significance. Finally, on the average, the observers were quite accurate in judging the depths of the four gaps. The mean (and $S D$ ) for the 14-cm gap was $14.7 \mathrm{~cm}(3.6 \mathrm{~cm})$; for the 46-cm gap, 51.1 (14.7); for the 92-cm gap, 95.3 (16.9); and for the 146-cm gap, 147.9 (53.3). It appears that the observers were aware of the actual depth of the gap.

In this as well as the remaining experiments, crossability judgments were obtained by using a method of

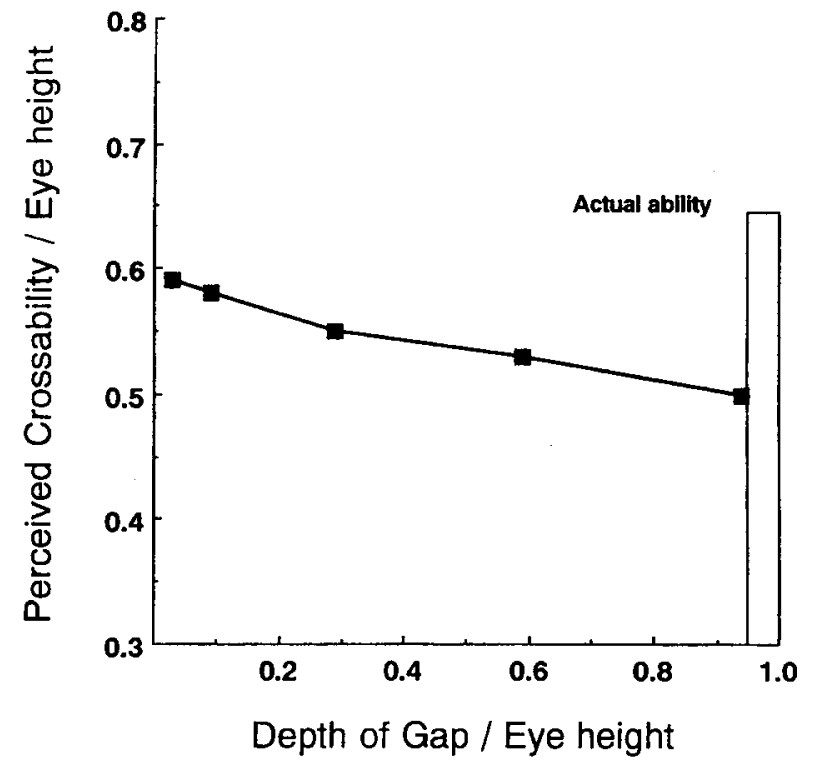

Figure 2. Experiment 1: mean perceived maximum crossable gap width as a function of depth of gap. The data for depth of gap/eye height equal to zero refer to judgments made when observers were standing on the ground. The bar graph indicates the mean actual capability. All measures are expressed in units of mean observer eye height.

limits in which the far side of the gap was moved either toward or away from the observer until the observer told the experimenter to stop. There is the possibility that the moving surface might not have appeared to afford stepping onto. As a consequence, participants might have become increasingly conservative in their judgments, allowing a greater margin of safety as gap depth increased. Two aspects of our procedure were intended to address this concern: First, the observers could make fine adjustments to the gap width after they told the experimenter to stop moving the apparatus; and second, they were encouraged to look at the apparatus while it was stationary, before indicating that they were satisfied with their estimate of gap width. Still, simply viewing the apparatus in motion might have caused these observers to increase the margin of safety in their judgments. A stronger means for assessing the effect of one's having viewed a moving apparatus is provided by the outcome of a replication of Experiment 1 with a methodology in which observers did not actually see the apparatus in motion. In this study, the observers faced away from the gap while the apparatus was being moved; they were instructed to turn around only when the apparatus was in position. For each gap width, the observers simply had to make a "yes"/"no" judgment about whether they could step over the gap in the prescribed manner. The results of this converging operation showed the same covariation between gap width and depth reported in this experiment. These data support our contention that the outcome of Experiment 1 was not simply an artifact of the observers' having seen the apparatus move. 
In summary, the results of Experiment 1 showed that perceived gap crossability covaried with gap depth. The experiments that follow were performed in an attempt to clarify the nature of this finding. Experiment 2 provided a converging method for verifying the covariation of perceived gap crossability and gap depth.

\section{EXPERIMENT 2}

This time, observers made their judgments of gap crossability in the dark. The gap was specified by luminescent stars that covered either the horizontal or the vertical surface of the opposing cliff. Both conditions provided the observers with information about the location of the edge. However, only the vertical condition provided information about the gap's depth. If perceived gap crossability covaries with gap depth, the observers' estimates of their maximum crossable gap width should be smaller when only the vertical surface is visible but not when only the horizontal surface is visible. In a third viewing condition, luminescent markers were placed on both the horizontal and the vertical surfaces. Since information was available about the vertical surface, we expected that the observers' judgments of the maximum crossable gap would be similar to their judgments when only the vertical surface was visible.

Method
Subjects
Sixteen female undergraduates at Miami University partici-
pated in the experiment to satisfy a course requirement. They were
asked to wear loose-fitting clothes. None of these women had par-
ticipated in Experiment 1 .
Apparatus
The apparatus from Experiment 1 was moved to a room that
could be effectively darkened. The movable side of the gap was
covered with burlap on which luminescent markers ("stars") were
mounted so that the markers were separated by roughly 9 cm hor-
izontally and vertically. One panel of burlap covered only the top,
while a second panel covered only the vertical wall of the gap. The
Styrofoam panel was not used to simulate the bottom of the gap.
Thus, the gap was $158 \mathrm{~cm}$ deep in all conditions.

\section{Viewing Conditions}

There were four viewing conditions. In Condition 1 , the horizontal-only condition, the panel of burlap with the luminescent markers was placed only on the top (horizontal surface) of the moving apparatus. A burlap panel without luminescent markers was placed on the vertical surface. Thus, the gap was depicted only by the top surface; the vertical surface appeared black and blended in with the surroundings. In Condition 2 , the vertical-only condition, the panel of burlap with the luminescent spots was placed only on the vertical surface of the moving apparatus. A panel without luminescent markers was placed on the top surface. The gap was depicted only by the vertical surface; the edge was covered with a luminescent line, but the horizontal surface was not. In Condition 3 , the horizontal and vertical condition, markers were placed on both the top and vertical panels. For the first three conditions, the edge of the horizontal surface was covered with a luminescent line and thus always visible. In Condition 4 , the light condition, the observers made their judgments with the room lights on. This condition was most nearly comparable to the 146- $\mathrm{cm}$-deep gap in Experiment 1 and thus provided a measure of the comparability of the viewing conditions in Experiments 1 and 2.

\section{Procedure}

Because we were primarily interested in comparing Conditions 1 and 2, only those conditions were counterbalanced. After the first two conditions were completed, Condition 3 was presented and followed by Condition 4 .

The task instructions and procedures were identical to those in Experiment 1. Four sets of ascending and descending trials were conducted under each viewing condition. The participants were asked to avert their gaze while the star-covered burlap panels were placed in position for the next viewing condition. After making the judgments in all four viewing conditions, the observers' actual stepping capabilities were measured using the same procedures followed in Experiment 1. Finally, we measured total standing height, eye height, and seated height.

\section{Results and Discussion}

Since only Conditions 1 and 2 were counterbalanced, a two-factor ANOVA (order and viewing condition) was performed to ascertain whether order had an effect on crossability judgments in the four viewing conditions. Order did not approach statistical significance, either as a main effect $[F(1,14)=1.13]$ or in its interaction with viewing condition $[F(3,42)=1.24]$. For each of the four viewing conditions, the mean crossability judgment for the two orders was within $2 \mathrm{~cm}$ of one another. Thus, it appears that the counterbalancing strategy employed did not have a meaningful effect on crossability judgments.

Condition 4 , in which observers viewed the gap in a fully lit room, was roughly comparable to the $146-\mathrm{cm}-$ deep gap in Experiment 1. As such, this condition provided a baseline for evaluating the comparability of the methods used in the first two experiments. The mean perceived maximum crossable gap was 0.50 of mean eye height in both Experiment 1 (146-cm gap) and Experiment 2 (Condition 4). Although a more extensive comparison of the outcome of the two experiments was not possible because Experiment 2 only had one gap depth, performance appeared to be comparable in the two experiments.

Figure 3 shows the mean perceived crossable gap (expressed in mean eye height) as function of viewing condition. A one-factor (condition), within-subjects ANOVA on Conditions 1-3 showed a significant main effect of viewing condition $[F(2,30)=6.21, p<.01]$. As expected, a Tukey HSD Test $(\alpha=.05)$ showed that estimates of the maximum crossable gap in Condition 1 ( $M=79.3 \mathrm{~cm}, 0.52$ eye height) were significantly greater than in Condition $2(M=73.0 \mathrm{~cm}, 0.48$ eye height $)$. The observers' judgments in the horizontal-only condition were quite accurate; they underestimated their actual stepping capabilities by $5 \%$. This outcome confirms the essential qualitative finding of Experiment 1, that judgments of the maximum crossable gap decrease as the gap becomes deeper. However, judgments in the verticalonly condition might have decreased because this condition lacked a clearly specified surface on which to step. Therefore, it is important to compare these data 


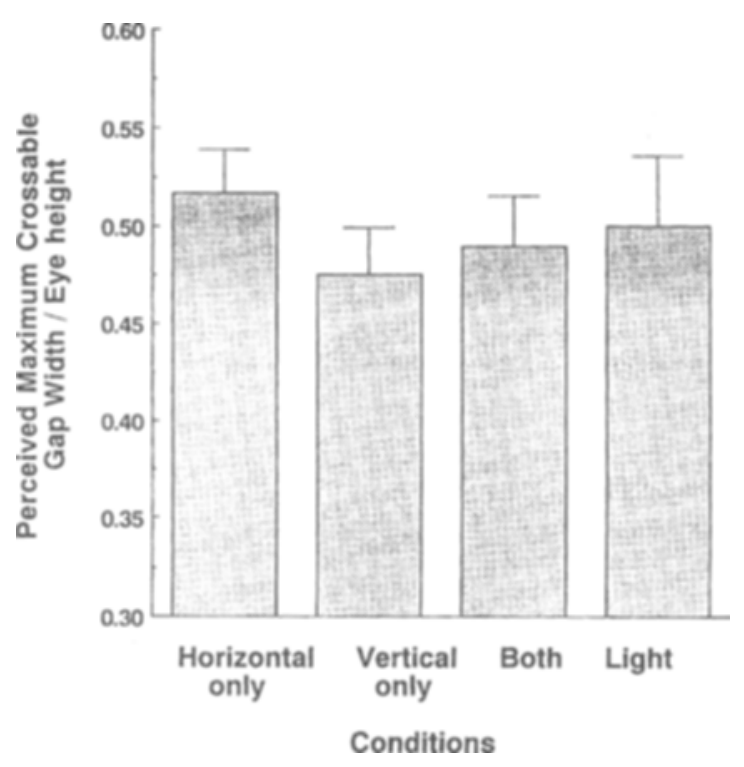

Figure 3. Experiment 2: mean perceived maximum crossable gap width in the four viewing conditions in units of mean observer eye height.

with those from the third condition, in which both surfaces were covered with luminescent spots. The mean perceived crossable gap width in Condition $3(75.2 \mathrm{~cm}$, 0.49 eye height) fell between the means for Conditions 1 and 2. Differences between Condition 3 and Conditions 1 and 2 did not reach the .05 level of significance. However, upon inspection of each participant's data, we found that some observers' judgments in Condition 3 were more similar to their judgments in the vertical-only condition than in the horizontal-only condition, while the reverse was true for other observers. During the debriefing sessions, several participants indicated that in Condition 3 they had been looking predominately at one of the surfaces. Since more than half of the participants indicated that they did not focus predominately on either the horizontal or the vertical surface, we did not attempt to correlate where participants were looking with their judgments in Condition 3. Still, the participants' comments during debriefing interviews led us to consider the possibility that direction of gaze may be a factor in judgments of perceived gap crossability.

There are at least two possible roles that direction of gaze might play in perceiving whether a gap is crossable. Gaze could operate strictly as an oculomotor factor in which movements of the eyes and head might figure in the interpretation of the optical stimulation. Alternatively, gaze might bear on the specific samples of the optic array that are obtained (Flach, 1990). Looking in different places reveals different information; when gaze is directed toward the top of the opposing cliff, observers appear to detect information specific to gap crossability (gap width) more readily than when they are looking down into the gap. While the remaining experiments were not intended to distinguish between these possibilities, gaze certainly affects sampling of the optic array, as well as the activity involved in the pickup of information about the gap: If a person is standing at the edge of a very deep gap, but is looking ahead toward the horizon (or slightly above it), her/his gaze would not permit detection of the full extent of the gap's depth. For this reason, we might expect judgments of crossability to be comparable to those for a gap of lesser depth. Although the gap exists physically, regardless of whether the observer is looking into the gap, from a purely optical standpoint, the vertical dimension of the opposing cliff must be in the observer's visual field. The extent to which the vertical surface is in the observer's visual field might determine the effect of gap depth on the observer's perception of crossability.

In Experiments 3 and 4, we examined whether direction of gaze can affect perception of gap crossability. Although we did not have equipment for monitoring where someone would be looking in the dark, our strategy was to instruct observers where to look in the gap and then measure the effect of direction of gaze on judgments of gap crossability. Will judgments of gap crossability covary with the physical depth of the gap or the observer's direction of gaze? If observers' judgments of the width of their maximum crossable gap size covary only with their direction of gaze, the outcomes of the two previous experiments may have been indicative more of where observers were looking than the physical depth of the gap per se. On the other hand, if the physical depth of the gap is the crucial determinant of observers' perceptual judgments of gap crossability, direction of gaze should have little effect.

\section{EXPERIMENT 3}

This experiment was designed to determine whether judgments of a gap's crossability are affected by the direction of the observer's gaze or the physical depth of the gap. Only one gap display was presented: Condition 3 from Experiment 2, in which both the horizontal and the vertical surfaces were visible. On each trial, the observers were instructed to look at one of two places on the movable apparatus, either at a landmark in the middle of the horizontal surface or a landmark on the vertical surface $120 \mathrm{~cm}$ below the top edge. If observers' direction of gaze is a crucial determinant of their perception, judgments of the widest crossable gap should be larger when observers are looking at the horizontal surface than the vertical surface.

\section{Method}

Eight advanced female undergraduates were presented with Condition 3 from Experiment 2, where luminescent markers were placed on both horizontal and vertical surfaces. On half the trials, observers were told to focus their gaze on a relatively large luminescent spot ( $3 \mathrm{~cm}$ in diameter) at the center of the horizontal surface; on the remaining trials, they were told to look at a similar luminescent spot placed on the vertical surface, $120 \mathrm{~cm}$ from the top edge. Instructions about where to look were given at the beginning of each trial. Each observer made 16 judgments under each gaze condition. A judgment was considered to be the average of an as- 
cending and a descending trial. The order of where observers directed their gaze was determined at random prior to the start of the experiment.

\section{Results and Discussion}

Each observer's perceived maximum crossable gap was expressed as a fraction of her eye height. The mean perceived maximum crossable gap width (and $S D$ s) was 0.52 eye height $(0.02)$ when observers were looking at the horizontal surface, and 0.48 eye height $(0.02)$ when they were looking at the vertical surface. The perceived maximum crossable gap was significantly greater when observers were looking at the horizontal surface than at the vertical surface $[F(1,7)=88.83, p<.01]$. This relative pattern of judgments was obtained for each of the 8 observers and reached statistical significance for each observer. Thus, the outcome of Experiment 3 supports the proposal that where observers look affects their perception of whether a gap affords stepping over.

\section{EXPERIMENT 4}

Another test of the gaze hypothesis is to vary where on the vertical surface observers are looking. Observers' estimates of their gap crossing capabilities should decrease as their gaze is directed further from the top edge.

\section{Method}

The apparatus in this experiment was identical to that used in Experiment 3, except that 3-cm-diameter luminescent circles were placed at four locations on the movable cliff: one marker was placed $3 \mathrm{~cm}$ from the edge on the horizontal surface, though for the purposes of our analysis, we treat it as being at $0-\mathrm{cm}$ depth; the remaining circles were placed 14,80 , and $140 \mathrm{~cm}$ from the top of the vertical surface. The placement of the markers was equidistant from either side of the cliff. These spots were intended as fixation points to which observers would direct their gaze. The remainders of both surfaces were covered with luminescent spots as in Experiment 3. A luminescent line was placed at the very bottom of the vertical surface.

Sixteen women undergraduates made perceptual judgments of the maximum gap width that they could step over. These observers were instructed to look at one of the fixation points. They made eight judgments at each fixation point-four ascending trials and four descending trials. After completing a block of eight judgments, the observers were instructed to attend to a different fixation point. The order in which the observers attended to the four fixation points was counterbalanced. All judgments were made in the dark. After the observers had completed their perceptual judgments, each observer's actual stepping capability was determined according to the method used in Experiment 1.

\section{Results and Discussion}

Figure 4 shows the mean perceived maximum crossable gap width for each of the four gaze locations, expressed as a percentage of their actual stepping capabilities. Judgments progressively decreased as gaze was directly deeper into the gap. A one-factor ANOVA revealed a significant main effect for gaze depth $[F(3,45)=$ $19.43, p<.001]$. A Tukey HSD test $(\alpha=.05)$ showed significant differences between the means for the $0-\mathrm{cm}$

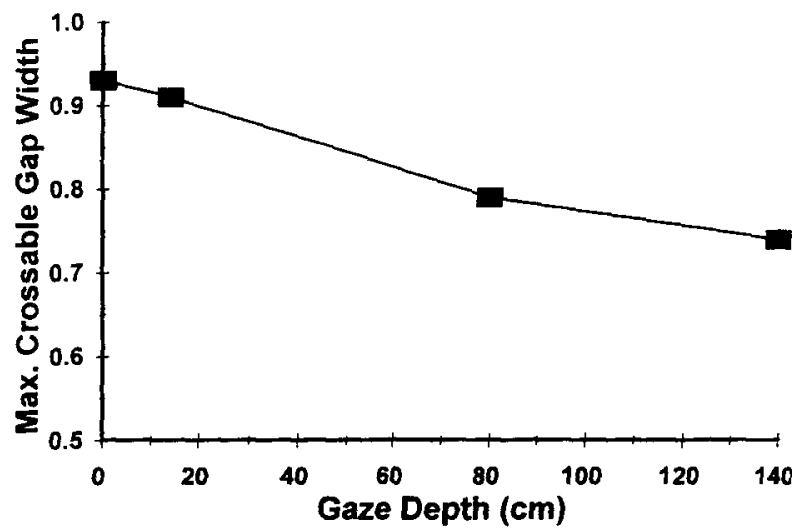

Figure 4. Experiment 4: mean perceived maximum crossable gap width expressed as a proportion of the mean actual stepping capability for four gaze depths. The data point for gaze depth $=0$ refers to the gaze circle located on the horizontal surface.

gaze depth (the fixation circle on the horizontal surface) and the two deepest gaze depths. Similarly, differences between the 14-cm gaze depth and the $80-\mathrm{cm}$ and $140-$ $\mathrm{cm}$ gaze depths and the $80-\mathrm{cm}$ and $140-\mathrm{cm}$ gaze depths were also significant. The difference between the $0-\mathrm{cm}$ and 14-cm gaze depths was not significant.

It is important to emphasize that the same optical display was used throughout the course of this experiment. That is, neither the gap's physical depth nor its optical depth was changed. The outcome was consistent with our proposal that perceived maximum crossable gap width is a function of where in the gap observers are looking. The current methodology does not eliminate the possibility that it may simply be harder to judge gap width when looking down into the gap. Perhaps it is more difficult to apprehend information about gap width when the gaze is directed downward. In the Appendix, we offer a proposal concerning the optical relations that specify gap width. A possible implication of this analysis is that it may be more difficult for observers to accurately apprehend the relevant optical relations that specify gap width when looking down into the gap than when looking toward the top of the opposite cliff.

\section{GENERAL DISCUSSION}

In the present investigation, we have examined whether a gap's depth is involved in determining whether a gap affords a safe crossing. The outcome was similar to that reported by Lock and Collett $(1979,1980)$ in their studies of how toads negotiated chasms. Experiments 1 and 2 demonstrated that human observers' judgments of gap crossability covaried with gap depth; judgments of the maximum crossable gap progressively decreased as gap depth increased. The outcomes of Experiments 3 and 4, however, clarified that finding: Judgments of gap crossability were affected not simply by the physical depth of the gap, but by where in the gap an observer's gaze was directed. Observers' estimates of their maximum cross- 
able gap width decreased when their gaze was directed toward the bottom of the gap; when looking at the ground plane (horizontal surface) on the opposing cliff, physical gap depth had little effect on observers' judgments.

\section{Criterion Shift}

Before examining the optical relations to which observers were attending as a consequence of their gaze, we must first consider the possibility that the obtained covariation of gap depth and perceived gap crossability reflects a progressive shift in observers' criteria for judging whether a gap is crossable. The effect of gaze observed in Experiments 3 and 4 is inconsistent with the proposal that observers became more conservative in their judgments or allowed a greater margin of safety as actual gap depth increased: the observers' judgments of a gap of constant physical depth varied with their direction of gaze; and, in each experiment, they were certainly aware of their physical distance from the floor (height of the platform) as well as the (optical) depth of the gap, even when they were looking at the horizontal surface. Finally, the outcome of a recent experiment (Mark, Jiang, \& Steinbach, 1994) provides an additional argument against basing an interpretation of our data on a criterion shift. When observers stood at the edge of the gap and estimated the width of the gap, the pattern of results was identical to what would be expected on the basis of the present study: When estimating the horizontal distance between the two vertical walls of the gap, the observers increasingly overestimated the horizontal distance as their gaze was directed progressively farther down into the gap. That is, the width of a gap was seen to increase when the observers looked down into the gap. (With respect to crossability judgments, perceiving the gap to be wider should result in a decrease in estimates of the maximum crossable gap width.) This suggests that the fundamental outcome of the present study was not strictly a consequence of considerations entailed in crossing the gap, since the possibility of crossing the gap was not mentioned to these observers. ${ }^{3}$

The same evidence that minimizes the involvement of a criterion shift in the present study also bears on a related interpretation based on the intrusion of analytic and emotional processes (Heft, 1993). As gap depth increased, observers may have become more conservative in assessing their capabilities, perhaps because of fear resulting from the increased risk to their safety. Thus, judgments of maximum crossable gap width should decrease. In this way analytic and emotional (fear) processes could have mediated judgments of whether a gap is crossable.

Again, however, the effect of (the direction of) gaze on judgments of gap crossability, as well as anecdotal evidence from participants, argue against this interpretation. Observers were certainly aware of the optical depth of the gap, regardless of whether they were looking at the horizontal or vertical surface of the opposing cliff. Yet direction of gaze overrode gap depth as a determinant of judgments of gap crossability. Observers were also well aware of the height (above the floor) of the platform on which they were standing, because they had to climb onto the apparatus. This knowledge, however, would have increased the level of emotional discomfort for all gap depths, regardless of gaze, since physical distance above the floor did not change throughout the experiment; thus, it would not account for the obtained covariation.

There are additional arguments against basing an interpretation of the present results on the intrusion of emotional or analytic processes. In Experiment 1, the optical depth was different in each condition; thus, it could be argued that observers increasingly underestimated their capabilities as the depth of the gap increased because they were more afraid when perceiving the deeper gap. But this argument does not hold for Experiment 4 , where the optical depth was held constant throughout the experiment. Finally, we also note that in our debriefing sessions, participants often indicated that they thought they could step farther when the optical depth was larger or they were looking at the vertical surface. For almost all the participants who expressed this expectation, their perceptual judgments reflected the opposite pattern; that is, their judgments were larger for shallower gaps or when they were looking at the horizontal surface.

In summary, the outcome of this investigation, to date, leads us to discount the proposal that either a criterion shift or emotional/analytic processes mediated perceptual judgments of gap crossability in the present experiment. Therefore, we turn to consider the optical relationships to which observers attend when making judgments about whether a gap is crossable.

\section{Optical Relations to Which Observers Attend}

An observer's action capabilities are a consequence of his/her own body size and biodynamic capabilities. Perceiving the distance from the edge of one cliff to the other, by itself, is not sufficient for an observer to determine whether the gap affords crossing; the observer has to determine how gap width is related to her/his own stepping capabilities.

A computational model might propose that this entails a comparison of perceived gap width and stored knowledge of one's own action capabilities; given the results of the present study, determination of perceived gap width, $W_{\mathrm{p}}$, would entail a computation involving both gap depth, $d$, and gap width, $w: W_{\mathrm{p}}=f(d, w)$. The simplest possible model would assign to gap depth a weight, $k$, based on the observer's direction of gaze: $W_{\mathrm{p}}=$ $f(k * d, w)$. Empirical data (past experience) might be used to assign a specific value to $k$.

Alternately, Gibson's (1979) ecological approach to the perception of affordances attempts to identify available information for a body-scaled measure of gap width. Geometric analyses have shown that surface 
height is specified with reference to each observer's eye height, an optically specified property (Lee, 1980; Sedgwick, 1973; Warren, 1984; Warren \& Whang, 1987). Other investigations have shown that observers actually use this available body-scaled information in making judgments about affordances for sitting and stair climbing (Mark, 1987), passing through apertures (Warren \& Whang, 1987), and reaching for objects (Carello, Grosofsky, Reichel, Solomon, \& Turvey, 1989). Although such geometric analyses do not constitute a completely adequate description of the relevant perceptual information (Konczak, Meeuwsen, \& Cress, 1992; Mark, Balliett, Craver, Douglas, \& Fox, 1990), they have provided a useful starting point for understanding how observers determine whether the environment will support their action capabilities. Thus, an ecological approach to determining whether a gap is crossable must identify optical relations that enable individuals to relate environmental properties to their own body scale and action capabilities. The Appendix presents a model of optical relationships that specify gap width (for gaps of various depths) with reference to the observer's eye height; we then offer a proposal for why perceived gap crossability covaries with gap depth as a function of direction of gaze.

The present study was not designed to compare the adequacy of computational and ecological approaches to the perception of affordances. It remains a formidable challenge to distinguish between these approaches, since the description of relevant optical relationships offered in the Appendix can be viewed as indicative of either what is detected directly by a "smart" perceptual mechanism (Runeson, 1977; an ecological interpretation) or in terms of a computational algorithm.

Finally, the appearance of a deep gap as being wider than a shallower gap of comparable width may disappear over time as a result of observers' exploratory actions. Mark (1987) changed subjects' leg lengths by having them wear blocks on their feet. Over a period of roughly $30 \mathrm{~min}$, observers were able to retune their perceptual boundary of the maximum surface height on which they could sit, to reflect their new capabilities. This occurred even though they were not given the opportunity to practice the relevant act or look at the blocks. Mark et al. (1990) showed that the errors disappeared when observers were allowed to perform appropriate exploratory actions. Bingham, Romack, and Stassen (1993) reported similar findings for reaching under displacement prisms. Although we did not find a consistent reduction in the underestimation of maximum crossable gap width over the course of the eight trials per subject, future studies should provide observers with additional trials and the opportunity to engage in exploratory activity needed to pick up information specifying gap width. In this regard, it would also be interesting to consider the possibility that people who view the world from and act in it at different heights, such as gymnasts and mountain climbers, might be less susceptible to this illusion.

\section{REFERENCES}

Bingham, G. P., Romack, J. L., \& Stassen, M. G. (1993). Optical information in visually guided reaching with perturbation of visual direction. In S. Valenti \& J. B. Pittenger (Eds.), Studies in perception and action (pp. 119-123). Hillsdale, NJ: Erlbaum.

Carello, C., Grosofsky, A., Reichel, F. D., Solomon, H. Y., \& TURVEY, M. T. (1989). Visually perceiving what is reachable. Ecological Psychology, 1, 27-54.

Flach, J. M. (1990). Control with an eye for perception: Precursors to an active psychophysics. Ecological Psychology, 2, 83-111.

GrBSON, J. J. (1979). The ecological approach to visual perception. Boston: Houghton-Mifflin.

HEFT, H. (1993). A methodological note on overestimates of reaching distance: Distinguishing between perceptual and analytical judgments. Ecological Psychology, 5, 255-271.

Konczak, J., Meeuwsen, H. J., \& Cress, M. E. (1992). Changing affordances in stair climbing: The perception of maximum climbability in young and older adults. Journal of Experimental Psychology: Human Perception \& Performance, 18, 691-697.

Kroemer, K. H. E., \& Price, D. L. (1982). Ergonomics in the office: Comfortable work stations allow maximum productivity. Industrial Engineering, 14, 24-32.

LEE, D. N. (1980). Visuo-motor coordination in space-time. In G. Stelmach \& J. Requin (Eds.), Tutorials in motor behavior (pp. 281-295). Amsterdam: North-Holland.

LOCK, A., \& COLLETT, T. (1979). A toad's devious approach to its prey: A study of some complex uses of depth vision. Journal of Comparative Physiology A, 131, 179-189.

Lock, A., \& ColleTt, T. (1980). The three-dimensional world of a toad. Proceedings of the Royal Society of London: Series B, 206, 481-487.

MARK, L. S. (1987). Eye height-scale information about affordance: A study of sitting and stair climbing. Journal of Experimental Psychology: Human Perception \& Performance, 13, 360-370.

Mark, L. S., Balliett, J., Craver, K., Douglas, S., \& Fox, T. (1990). What an actor must do in order to perceive the affordance for sitting. Ecological Psychology, 2, 325-365.

MARK, L. S., JiANG, Y., \& STEINBACH, S. (1994). The pickup of information about what a gap affords. Manuscript in preparation.

RUNESON, S. (1977). On the possibility of "smart" perceptual mechanisms. Scandinavian Journal of Psychology, 18, 172-179.

SEDGWICK, H. A. (1973). The visible horizon: A potential source of visual information for the perception of size and distance (Doctoral dissertation, Cornell University, 1973). Dissertation Abstracts, 34, 1301B-1302B (University Microfilms No. 73-22530).

SEDGWICK, H. A. (1980). The geometry of spatial layout in pictorial representation. In $M$. Hagen (Ed.), The perception of pictures (pp. 33-90). New York: Academic Press.

WARREN, W. H. (1984). Perceiving affordances: Visual guidance of stair climbing. Journal of Experimental Psychology: Human Perception \& Performance, 10, 683-703.

WARREN, W. H., \& WHANG, S. (1987). Visual guidance of walking through apertures: Body scaled information for affordances. Journal of Experimental Psychology: Human Perception \& Performance, 13, 371-383.

\section{NOTES}

1. Although different types of actions may be used in crossing a gap (e.g., leaping, jumping), in the present study we focus on an action that is best described as stepping-at all times the actor has at least one foot in contact with the ground plane. (The act is defined under the Procedure for Experiment 1.) As the gap's width increases, the limit on the actor's ability to step across the gap is reached. At some critical gap width, the actor will have to shift to another style of crossing--say, leaping-in order to cross the gap. In each of the present experiments, the observers were asked to estimate the maximum gap width that they were confident they could step across safely. 
2. Judgments of maximum crossable distance on the ground were correlated with anthropometric measures of each observer's leg length and eye height. A stronger correlation was observed for eye height $(r=.61)$ than for leg length $(r=.28)$. For this reason, perceptual judgments throughout the present study were expressed in terms of the mean eye height. Eye height has been implicated as an optically specified, body-scaled measure of environmental properties, such as surface height (Mark, 1987; Sedgwick, 1973; Warren \& Whang, 1987; see also the Appendix). That eye height was more strongly correlated with perceptual judgments than leg length may indicate that perceptual judgments about distance are based on eye height-scaled information. (See the Appendix.)

3. The arguments against a criterion shift as accounting for the covariation of gap depth and perceived crossability do not rule out the possibility that in actually stepping across a gap observers might increase their margin of safety as gap depth increases. This could be realized by trying to achieve a greater distance between the placement of the lead foot and the edge of the gap or by changing the style of action used to cross deeper gaps-say, from stepping to leaping. In the present study, the observers, who were looking at the horizontal surface, still tended to underestimate their actual capabilities by $5 \%-10 \%$ for all gap depths. This underestimation may be indicative of a margin of safety. Similar findings have been reported by Mark (1987), in an investigation of the maximum height of a surface onto which subjects could climb or on which they could sit. Warren and Whang (1987), in their study of passing through apertures, found that observers rotated their shoulders in order to pass through an aperture when the ratio of aperture width to shoulder width approached 1.20; this ratio allowed a comfortable margin of safety so that actors would avoid touching the surface of the aperture.

\section{APPENDIX \\ A Geometric Model for Relating Gap Width to the Observer's Body Scale (Eye Height) Based on Sedgwick's (1973) Analysis of Surface Height}

The ecological approach to the perception of an affordance attempts to identify optical relationships that specify relevant environmental properties with reference to the observer's own body (action) scale. Sedgwick (1973) has identified one such optically specified body-scaled unit, eye height, which refers to the distance between the point of observation and the ground plane. The goal of this analysis is to derive an equation in which gap width, $w$, scaled in terms of the observer's eye height, $h$ (i.e., $w / h$ ), is expressed in terms of available optical relationships (visual angles). We will begin by identifying such optical specifications for (1) the distance a person can step on the ground (i.e., a gap whose depth $=0$ ), (2) the depth of a gap, and (3) the width of a gap of some finite depth.

In the following discussion (see Figures $\mathrm{A} 1$ and $\mathrm{A} 2$ ), $h$ is the perceiver's eye height; $d$ is the depth of the gap; $w$ is the actual width of the gap; $w_{\mathrm{g}}$ is the physical maximum distance a person can step on the ground; $w_{\mathrm{e}}$ is the physical maximum crossable gap width for a person standing at the edge of the gap; $w_{e}^{\prime}$ is the perceived maximum crossable gap width for a person standing at the edge of the gap; the angle $\theta$ is formed by the horizon, point of observation, and the maximum crossable point on the ground; and angle $G$ is the angle formed by the horizon, point of observation, and a point on the vertical wall of the opposing cliff to which the observer's gaze is directed.

Sedgwick (1973) has shown that the horizon-ratio relationship specifies surface height in terms of each observer's eye height. The same relationship can also specify the distance a person is able to step (Figure A1, top panel):

$$
w_{\mathrm{g}} / h=1 / \tan \theta .
$$

This equation shows that the physical distance a person can step is optically specified in terms of the person's eye height.
Some critical value of angle $\theta$ specifies the maximum width of a gap that a particular observer is able to step over. Thus, the maximum crossable gap width can be optically specified with reference to the observer's eye height.

For a person standing at the edge of the gap (Figure A1, bottom panel), gap depth can be specified in terms of two angles, $G_{\mathrm{e}}$ and $\theta_{\mathrm{e}}$ :

$$
\begin{gathered}
w /(h+d)=1 / \tan G_{\mathrm{e}}, \\
w / h=1 / \tan \theta_{\mathrm{e}} .
\end{gathered}
$$

Combining Equations $\mathrm{A} 2$ and $\mathrm{A} 3$ to eliminate $w$, we obtain, when $d>0$ :

$$
d / h=\left(\tan G_{\mathrm{e}}-\tan \theta_{\mathrm{e}}\right) / \tan \theta_{\mathrm{e}}
$$

Equation A4 shows that the physical depth of the gap is specified with reference to the observer's eye height.

How is gap width specified when $d>0$ ? When the observer's gaze is directed toward the horizontal surface or near the brink of the opposing cliff so that the depth is not in the observer's field of view, we propose that gap width, $w_{\mathrm{e}}$, is specified by

$$
w_{\mathrm{e}} / h=w_{\mathrm{g}} / h=1 / \tan \theta=1 / \tan \theta_{\mathrm{e}} .
$$

Gap depth can be ignored, since little of the vertical surface is within the field of view. The outcome of Experiment 3 supports this assumption; when gaze was directed toward the horizontal surface, judgments of the maximum crossable gap
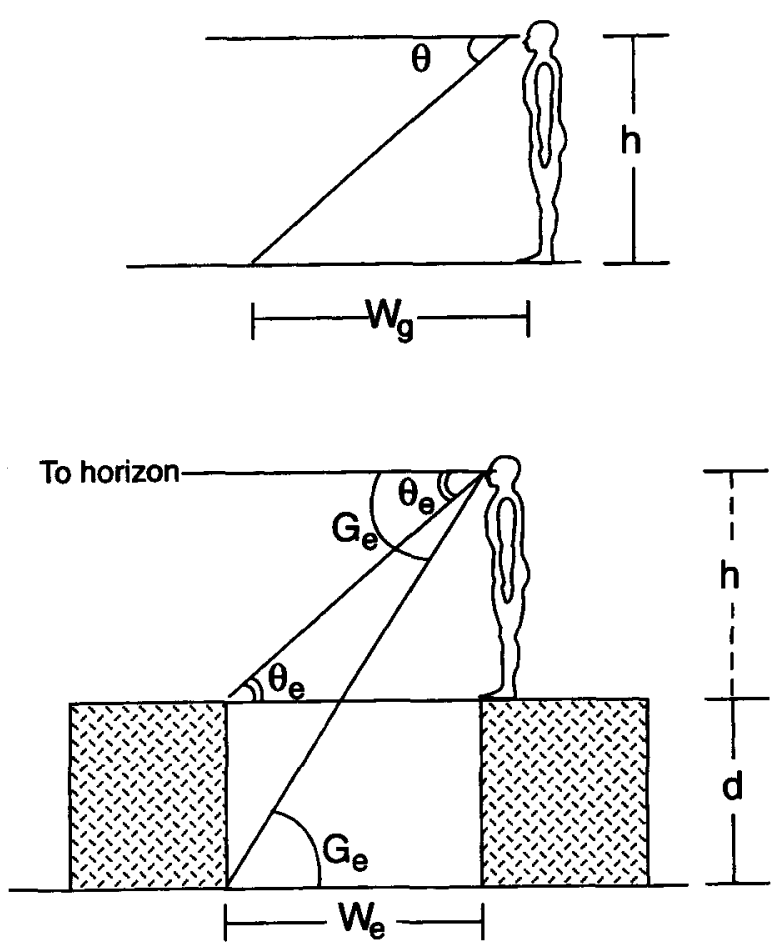

Figure A1. Top: geometric relations specific to the observer's maximum crossable distance on the ground plane for an observer of eye height $h: w_{\mathrm{g}} / h=1 / \tan \theta$. Some critical value of $\theta$ specifies the limits of the observer's capability for crossing the gap. Bottom: geometric relations specific to the depth of a gap for an observer of eye height $\boldsymbol{h}: d / \boldsymbol{h}=\left(\tan G_{\mathrm{e}}-\tan \theta_{\mathrm{e}}\right) / \tan \theta_{\mathrm{e}}$. Geometric analysis for perceived crossable gap size for an observer with eye height $h$, and gap with depth $\boldsymbol{d}$. See the Appendix for details. 


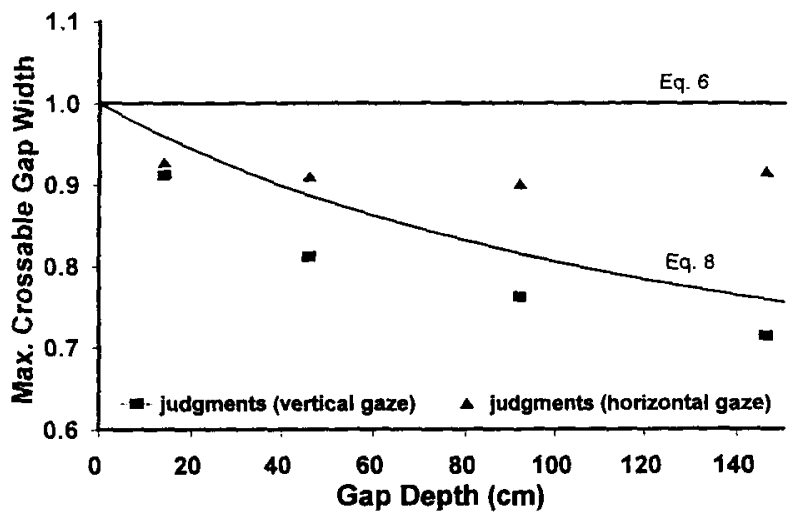

Figure A2. Mean perceived maximum crossable gap width expressed as a proportion of the mean actual stepping capability for four gap depths and two gaze directions (horizontal surface and bottom of the vertical surface). The line labeled as Equation 6 depicts predictions of the model of the information available about gap width for gaps of different depths; the trend of the data points obtained under the horizontal gaze viewing condition closely approximates this function. The underestimation may reflect the observer's margin of safety. The line labeled as Equation 8 shows the predictions of the model in Equation 8 for why observers tend to underestimate their capabilities as gap depth increases.

width were significantly greater than judgments made while looking at the vertical surface, and they were comparable to those made when standing on the floor in Experiment 1.

When the observer's gaze is directed toward the vertical surface at depth, $d$, from the top of the gap, $w$ is specified by (Figure $\mathrm{A} 1$, bottom panel)

$$
w_{\mathrm{e}} /(h+d)=1 / \tan G_{\mathrm{e}} .
$$

The expression $h+d$ refers to the observer's new eye height, the distance along the vertical axis between the point of observation and the location in the gap to which gaze is directed. Thus, in principle, there are optical relations that specify gap width, even when $d>0$. If observers can detect their new eye height as their direction of gaze is directed toward the vertical surface of the gap, judgments of gap width should be accurate. Why, then, do observers increasingly underestimate their maximum crossable gap width as their gaze is directed increasingly farther down into the gap?
We propose that the obtained underestimation is at least partially due to difficulty in registering subtle optical relations that specify an observer's new eye height. Mark (1987, Experiment 1) has shown that observers consistently underestimate their eye height as distance from the surface being viewed increases. Moreover, at a gap width comparable to the perceived maximum crossable gap width in the present study, and as gap depth increases beyond $0.5 h$, the rate of change in the gradient density becomes very small, making it difficult for observers to detect information about change in depth. In view of how little is known about the specification of eye height when the horizon is not in the field of view (see Sedgwick, 1973,1980 ), it is premature to offer quantitative predictions based on the underestimation of eye height.

Figure A2 presents predictions of the above analysis of the optical information about gap crossability: (1) If observers use the information about gap width identified in Equation A6, in which their eye height is correctly registered, judgments of maximum crossable gap width should remain constant with changes in gap depth. (2) If observers use the optical relations ( $1 / \tan G_{e}$ ) identified in Equation A6, but substitute the distance between the point of observation and their feet as their eye height $(h)$, rather than $h+d$, we obtain the following:

$$
w_{\mathrm{e}} / h=1 / \tan G_{\mathrm{e}} \text {. }
$$

On the basis of Equation A7, judgments covary with gap depth. However, Equation A7 predicts a greater underestimation than is actually obtained (Figure A2). It appears that observers apprehend their eye height as something between $h$ and $h+d$. As a first attempt to model the perceptual data when the observers' gaze was directed to the vertical surface, we combined Equations $\mathrm{A} 5$ and $\mathrm{A} 7$, assigning the two optical relations $1 / \tan \theta_{\mathrm{e}}$ and $1 / \tan G_{\mathrm{e}}$ equal weights $\left(r^{2}=.85\right)$ :

$$
w_{\mathrm{e}}^{\prime} / h=0.5\left[\left(1 / \tan \theta_{\mathrm{e}}\right)+\left(1 / \tan G_{\mathrm{e}}\right)\right] .
$$

Consistent with the proposal that eye height is misregistered, Equation A8 assumes that observers do not register their new eye height accurately. However, Equation A8 makes the additional assumption that when gaze is directed into the gap, perceived gap width is based on a combination of visual angles $\theta_{\mathrm{e}}$ and $G_{\mathrm{e}}$.

(Manuscript received September 1, 1993; revision accepted for publication June 19, 1994.) 